\title{
ON THE STRUCTURE OF CERTAIN BASIC SEQUENCES ASSOCIATED WITH AN ARITHMETIC FUNCTION
}

\author{
by DONALD L. GOLDSMITH $\dagger$
}

(Received 23rd July 1970)

\section{Introduction}

We have previously studied in some detail the multiplicative properties of a given arithmetic function $f$ with respect to a fixed basic sequence $\mathscr{B}$ (see, for example, (1), (2)). We investigate here the structure of $M(f)$, the collection of all basic sequences $\mathscr{B}$ such that $f$ is multiplicative with respect to $\mathscr{B}$, and in particular we focus our attention on the maximal members of $M(f)$. Our principal result will be a proof that each maximal member of $M(f)$ contains the same set of type II primitive pairs. Moreover, we will give a simple criterion for determining, in terms of the behaviour of $f$, whether or not a particular primitive pair $(p, p)$ is in any (and therefore every) maximal member of $M(f)$.

A basic sequence $\mathscr{B}$ is a set of pairs $(a, b)$ of natural numbers for which

(1) $(1, k) \in \mathscr{B}, \quad k=1,2, \ldots$;

(2) if $(a, b) \in \mathscr{B}$, then $(b, a) \in \mathscr{B}$;

(3) $(a, b c) \in \mathscr{B}$ if and only if $(a, b) \in \mathscr{B}$ and $(a, c) \in \mathscr{B}$.

If $\Phi$ is any collection of pairs of natural numbers, we set $\Gamma[\Phi]=\bigcap \mathscr{E}$, where the intersection is taken over all basic sequences $\mathscr{E}$ which contain $\Phi$. If $\Phi=\varnothing$, then $\Gamma[\Phi]=\mathscr{S}$, where $\mathscr{S}$ is the basic sequence consisting only of all pairs of the form $(1, k)$ and $(k, 1)(k=1,2, \ldots)$. A pair $(a, b)$ of natural numbers is called a primitive pair if both $a$ and $b$ are primes. It is of type $I$ if $a \neq b$, type II if $a=b$.

We assume, in order to avoid trivial situations, that no arithmetic function is eventually zero. An arithmetic function $f$ is said to be multiplicative with respect to a basic sequence $\mathscr{B}$ if $f(m) f(n)=f(m n)$ for all $(m, n) \in \mathscr{B}$. The set of all arithmetic functions which are multiplicative with respect to $\mathscr{B}$ is denoted by $M(\mathscr{B})$, and for a given arithmetic function $f, M(f)$ represents the set of all basic sequences $\mathscr{B}$ for which $f \in M(\mathscr{B})$.

A basic sequence $\mathscr{B}$ is a maximal member of $M(f)$ if $f \in M(\mathscr{B})$, but $\left.f \notin M(\mathscr{B})^{\prime}\right)$ for any basic sequence $\mathscr{B}^{\prime}$ which properly contains $\mathscr{B}$. The set of maximal members of $M(f)$ is denoted by $M^{*}(f)$. We prove in Lemma 2.1 that every member of $M(f)$ is contained in a member of $M^{*}(f)$, hence for the study of $M(f)$ it is sufficient to confine our attention to $M^{*}(f)$.

$\dagger$ This research was supported in part by Western Michigan University under a Faculty Research Fellowship.

E.M.S. -U 


\section{The structure of $M^{*}(f)$}

We will show first that for $M^{*}(f)$ to be non-empty it is both necessary and sufficient that $f(1)=1$. This result is an easy consequence of

Lemma 2.1. If $\mathscr{B}_{0} \in M(f)$, then there is a basic sequence $\mathscr{B}^{\prime}$ in $M^{*}(f)$ such that $\mathscr{B}_{0} \subset \mathscr{B}^{\prime}$.

Proof. The proof of the lemma will depend on Tukey's Lemma: Every non-empty family of sets of finite character has a maximal member. (A family $\mathfrak{A}$ of sets is of finite character provided $A \in \mathfrak{A}$ if and only if every finite subset of $A$ is in $\mathfrak{U} . \quad A$ is a maximal member of $\mathfrak{A}$ if $A \in \mathfrak{A}$ and if there is no member $A^{\prime}$ of $\mathfrak{A}$ such that $A^{\prime}$ properly contains $A$.)

Let $\Phi_{0}$ be the set of primitive pairs in $\mathscr{B}_{0}$ (take $\Phi_{0}=\varnothing$ if $\mathscr{B}_{0}=\mathscr{S}$ ) and define $\mathscr{A}$ to be the family of all sets $\Phi$ of primitive pairs for which

$$
f \in M\left(\Gamma\left[\Phi \cup \Phi_{0}\right]\right) \text {. }
$$

We will show that $\mathfrak{A}$ is non-empty and of finite character. By Tukey's Lemma, $\mathfrak{A}$ will then contain a maximal member, say $\Phi^{\prime}$, and it is clear that the basic sequence $\mathscr{B}^{\prime}=\Gamma\left[\Phi^{\prime}\right]$ will satisfy the requirements of Lemma 2.1 .

$\mathfrak{A}$ is non-empty, since $f \in M\left(\mathscr{B}_{0}\right)=M\left(\Gamma\left[\phi \cup \Phi_{0}\right]\right)$, so $\phi \in \mathfrak{A}$.

Suppose that $\Phi \in \mathfrak{U}$ and that $\Psi$ is any finite subset of $\Phi$. Since

$$
f \in M\left(\Gamma\left[\Phi \cup \Phi_{0}\right]\right)
$$

and since $\Gamma\left[\Psi \cup \Phi_{0}\right] \subset \Gamma\left[\Phi \cup \Phi_{0}\right]$, it follows that $f \in M\left(\Gamma\left[\Psi \cup \Phi_{0}\right]\right)$; hence $\Psi \in \mathfrak{H}$.

Conversely, let $\Phi$ be a set of primitive pairs and suppose $\Psi \in \mathfrak{A}$ for every finite subset $\Psi$ of $\Phi$. Let $(a, b)$ be any pair in $\Gamma\left[\Phi \cup \Phi_{0}\right]$ and let $\Psi_{1}$ be the set of all primitive pairs $(p, q)$ for which $p \mid a$ and $q \mid b$. Clearly $\Psi_{1} \subset \Phi \cup \Phi_{0}$. Now set

$$
\Psi=\Psi_{1} \cap \Phi \text {. }
$$

Then $\Psi$ is a finite subset of $\Phi$, so by assumption $\Psi \in \mathfrak{A}$. Therefore

But

$$
f \in M\left(\Gamma\left[\Psi \cup \Phi_{0}\right]\right) \text {. }
$$

$$
\begin{aligned}
(a, b) & \in \Gamma\left[\Psi_{1}\right]=\Gamma\left[\Psi_{1} \cap\left(\Phi \cup \Phi_{0}\right)\right] \\
& =\Gamma\left[\Psi \cup\left(\Psi_{1} \cap \Phi_{0}\right)\right] \subset \Gamma\left[\Psi \cup \Phi_{0}\right],
\end{aligned}
$$

so $f(a b)=f(a) f(b)$. It follows that $f \in M\left(\Gamma\left[\Phi \cup \Phi_{0}\right]\right)$ and so $\Phi \in \mathfrak{H}$.

Thus $\mathfrak{A}$ is of finite character and the proof is complete.

The result of Lemma 2.1 provides the basis for an easy proof of

Theorem 2.2. $M^{*}(f) \neq \varnothing$ if and only if $f(1)=1$.

Proof. If $f(1)=1$, then $f \in M(\mathscr{S})$, so $\mathscr{S} \in M(f)$. By Lemma 2.1 there is a basic sequence $\mathscr{B} \mathscr{B}^{\prime}$ in $M^{*}(f)$, therefore $M^{*}(f) \neq \varnothing$. 
On the other hand, if $f(1) \neq 1$, then $f \notin M(\mathscr{B})$ for any basic sequence $\mathscr{B}$; that is, $M(f)=\varnothing$. But since $M^{*}(f) \subset M(f)$, we have $M^{*}(f)=\varnothing$.

The next theorem will yield not only the previously mentioned result about the type II primitive pairs in the members of $M^{*}(f)$, but also will provide information about the distribution of the type I primitive pairs. We will use the following notation: For a given basic sequence $\mathscr{B}$ and a given prime $p$, we define

$$
C_{\mathscr{B}}(p)=\{q \mid q \text { prime, }(p, q) \in \mathscr{B}\} .
$$

Theorem 2.3. If

$$
f\left(p^{a} q^{b}\right)=f\left(p^{a}\right) f\left(q^{b}\right)
$$

for all natural numbers $a$ and $b$, then the primitive pair $(p, q)$ is contained in every basic sequence $\mathscr{B}$ in $M^{*}(f)$ for which $C_{\mathscr{B}}(p)=C_{\mathscr{B}}(q)$.

Proof. Suppose that $\mathscr{B} \in M^{*}(f)$ and $C_{\mathscr{D}}(p)=C_{\mathscr{O}}(q)$, but that the primitive pair $(p, q) \notin \mathscr{B}$. Define the basic sequence $\mathscr{B}^{\prime}$ by

$$
\mathscr{B}^{\prime}=\Gamma[\mathscr{B} \cup(p, q)] \text {. }
$$

Since $\mathscr{B}^{\prime}$ properly contains $\mathscr{B}, f \notin M\left(\mathscr{B}{ }^{\prime}\right)$.

Any element in $\mathscr{B}^{\prime}-\mathscr{B}$ must be of the form $\left(p^{a} v, q^{b} w\right)$ or $\left(q^{b} w, p^{a} v\right)$ where $p \nmid v$ and $q \nmid w$, where $a \geqq 1$ and $b \geqq 1$, where $v$ and $w$ are divisible only by primes from $C_{\mathscr{B}}(p)\left(=C_{\mathscr{B}}(q)\right)$, and where $(v, w) \in \mathscr{B}$. Since $(p, v),(p, w),(q, v)$, $(q, w)$ are all in $\mathscr{B},\left(p^{a} q^{b}, v w\right)$ is also in $\mathscr{B}$. Therefore, for any pair $\left(p^{a} v, q^{b} w\right)$ in $\mathscr{B}^{\prime}-\mathscr{B}$, we have

$$
\begin{gathered}
f\left(p^{a} q^{b} v w\right)=f\left(p^{a} q^{b}\right) f(v w)=f\left(p^{a} q^{b}\right) f(v) f(w), \\
f\left(p^{a} v\right)=f\left(p^{a}\right) f(v), \quad f\left(q^{b} w\right)=f\left(q^{b}\right) f(w) .
\end{gathered}
$$

On the other hand, since $f \notin M\left(\mathscr{B}^{\prime}\right)$ there is a pair $(m, n)$ in $\mathscr{B}^{\prime}-\mathscr{B}$ for which $f(m n) \neq f(m) f(n)$. So for some choice of $a, b, v, w$ we have

$$
f\left(p^{a} q^{b} v w\right) \neq f\left(p^{a} v\right) f\left(q^{b} w\right) .
$$

For this choice of $a, b, v, w$, relations (2.2), (2.3), (2.4) yield

$$
f\left(p^{a} q^{b}\right) f(v) f(w) \neq f\left(p^{a}\right) f\left(q^{b}\right) f(v) f(w),
$$

and so $f\left(p^{a} q^{b}\right) \neq f\left(p^{a}\right) f\left(q^{b}\right)$.

Corollary 2.4. If $\mathscr{B}$ and $\mathscr{B}^{\prime}$ are members of $M^{*}(f)$ and the primitive pair $(p, q) \in \mathscr{B}^{\prime}-\mathscr{B}$, then $C_{\mathscr{B}}(p) \neq C_{\mathscr{B}}(q)$.

A prime $p$ is said to be isolated from a basic sequence $\mathscr{B}$ if $C_{\mathscr{B}}(p)=\varnothing$.

Corollary 2.5. If $\mathscr{B}$ and $\mathscr{B}^{\prime}$ are members of $M^{*}(f)$ and the primitive pair $(p, q) \in \mathscr{B}^{\prime}$, then either $p$ or $q$ (or both) is not isolated from $\mathscr{B}$.

If we set $p=q$ in Theorem 2.3 we get the desired characterization of the type II primitive pairs in the members of $M^{*}(f)$. 
Theorem 2.6. Every basic sequence in $M^{*}(f)$ contains the same set of type II primitive pairs, namely, those pairs $(p, p)$ for which $p$ satisfies

$$
f\left(p^{n}\right)=f^{n}(p) \quad(n=1,2, \ldots)
$$

Proof. If $f(1) \neq 1$, then $M^{*}(f)$ is empty and there is nothing to prove. Otherwise, suppose $f(1)=1$ and $(p, p) \in \mathscr{B}_{0}$ for some basic sequence $\mathscr{B}_{0}$ in $M^{*}(f)$. Then $\left(p^{a}, p^{b}\right) \in \mathscr{B}_{0}$ and, since $f \in M\left(\mathscr{B}_{0}\right),(2.1)$ holds (with $p=q$ ). Since $p=q$ implies $C_{\mathscr{B}}(p)=C_{\mathscr{B}}(q)$ for every basic sequence $\mathscr{B}$, it follows from Theorem 2.3 that $(p, p)$ is in every member of $M^{*}(f)$. Thus the members of $M^{*}(f)$ contain the same type II primitive pairs, and these are clearly just the pairs $(p, p)$ such that $p$ satisfies $(2.5)$.

\section{An example}

In the previous section we investigated the structure of $M^{*}(f)$, the set of maximal members of $M(f)$. We may now ask the following question: Suppose the requirement that $f$ be multiplicative with respect to $\mathscr{B}$ is replaced by the less stringent requirement that $f$ be non-singular with respect to $\mathscr{B}$ (and, accordingly, $M(f)$ is replaced by the larger collection $N(f)$, consisting of those basic sequences $\mathscr{B}$ such that $f$ is non-singular with respect to $\mathscr{B})$. What can be said about the structure of $N^{*}(f)$, the maximal members of $N(f)$ ? We will show here (in Example 3.1) that there are arithmetic functions $f$ for which $N(f)$ has no maximal members, even though $f(1)=1$ and $N(f)$ is not empty (compare this with Lemma 2.1 and Theorem 2.2). Thus while the requirement $f(1)=1$ is enough to guarantee that $N(f)$ is not empty, it is not sufficient to ensure that $N^{*}(f)$ is not empty.

As a matter of convenience we repeat here the pertinent definitions (see (1) for a more complete exposition). For an arithmetic function $f$ and a pair $(m, n)$ of natural numbers we set

$$
\alpha_{f}(m, n)=\left\{\begin{array}{cl}
\frac{f(m) f(n)-f(m n)}{|f(m) f(n)|+|f(m n)|} & \text { if }|f(m) f(n)|+|f(m n)|>0, \\
0 & \text { if } f(m) f(n)=f(m n)=0 .
\end{array}\right.
$$

We say that the index of multiplicativity of $f$ with respect to the basic sequence $\mathscr{B}$ exists and has the value $I(f, \mathscr{B})$ provided

$$
\lim _{k \rightarrow \infty} \alpha_{f}\left(m_{k}, n_{k}\right)=I(f, \mathscr{B})
$$

for every sequence of pairs $\left\{\left(m_{k}, n_{k}\right)\right\}_{k=1}^{\infty}$. contained in $\mathscr{B}$ for which

$$
\lim _{k \rightarrow \infty} m_{k} n_{k}=\infty \text {. }
$$

We say $f$ is non-singular with respect to $\mathscr{B}$ if $I(f, \mathscr{B})$ exists and has the value zero, and we denote the set of all functions which are non-singular with respect 
to $\mathscr{B}$ by $N(\mathscr{B})$. We denote by $N(f)$ the set of basic sequences $\mathscr{B}$ for which $f \in N(\mathscr{B})$, and by $N^{*}(f)$ the set of maximal members of $N(f)$.

For any arithmetic function $f$, if $f \in M(\mathscr{B})$ then clearly $f \in N(\mathscr{B})$. Therefore

$$
M(f) \subset N(f) .
$$

The above inclusion may or may not be proper; it is easy to find functions satisfying either alternative.

Example 3.1. Define $f$ by

$$
f(1)=1, f(p)=1 \text { ( } p \text { prime }), f(n)=0 \text { otherwise. }
$$

We note that $M(f)=M^{*}(f)=\{\mathscr{S}\}$, for if $(p, q)$ is any primitive pair, then $f(p q)=0 \neq 1=f(p) f(q)$.

The proof that $N^{*}(f)$ is empty will depend on the fact that $f \notin N(\mathscr{B})$ for any basic sequence $\mathscr{B}$ which contains infinitely many type II primitive pairs. For suppose the sequence of primitive pairs $\left\{\left(p_{n}, p_{n}\right)\right\}_{n=1}^{\infty}$ is in $\mathscr{B}$, where we may suppose that $p_{1}<p_{2}<\ldots$. Then

$$
\alpha_{f}\left(p_{n}, p_{n}\right)=\frac{f^{2}\left(p_{n}\right)-f\left(p_{n}^{2}\right)}{f^{2}\left(p_{n}\right)+f\left(p_{n}^{2}\right)}=1 .
$$

Therefore $\lim _{n \rightarrow \infty} \alpha_{f}\left(p_{n}, p_{n}\right) \neq 0$ and so $f \notin N(\mathscr{B})$.

Suppose now that $\mathscr{B}$ is any member of $N(f)$ (these exist: $\mathscr{S}$, for example, or any basic sequence generated by a finite number of type II primitive pairs). By the remark above, $\mathscr{B}$ can contain only finitely many type II primitive pairs. Suppose then that $(q, q) \notin \mathscr{B}$ for some prime $q$ and let

$$
\mathscr{B}^{\prime}=\Gamma[\mathscr{B} \cup(q, q)] .
$$

Since $\mathscr{B}^{\prime}$ properly contains $\mathscr{B}$, it is sufficient to prove that $f \in N\left(\mathscr{B}^{\prime}\right)$.

Let $\left\{\left(m_{v}, n_{v}\right)\right\}_{v=1}^{\infty}$ be any sequence of pairs in $\mathscr{B}^{\prime}$ for which $m_{v}>1, n_{v}>1$, $m_{v} n_{v} \rightarrow \infty$. Split the sequence $\left\{m_{v}, n_{v}\right\}$ into two parts: (1) those $\left(m_{v}, n_{v}\right)$ in $\mathscr{B}$, (2) those $\left(m_{v}, n_{v}\right)$ in $\mathscr{B}^{\prime}-\mathscr{B}$.

For (1) we have immediately $\lim \alpha_{f}\left(m_{v}, n_{v}\right)=0$ since $f \in N(\mathscr{B})$.

Suppose then that $\left(m_{v}, n_{v}\right) \in \stackrel{\nu \rightarrow \infty}{\mathscr{B}^{\prime}}-\mathscr{B}$ and $m_{v} n_{v}>q^{2}$. Then

$$
m_{v}=x_{v} q^{a_{v}}, n_{v}=y_{v} q^{b_{v}} \text { with } a_{v} \geqq 1, b_{v} \geqq 1,
$$

and either $m_{v}>q$ or $n_{v}>q$, say $m_{v}>q$. If $m_{v}$ were prime, then $m_{v}=q$ by (3.1). But $m_{v}>q$, so $m_{v}$ is not prime and $f\left(m_{v}\right)=0$. Hence $f\left(m_{v}\right) f\left(n_{v}\right)=0$. On the other hand, $m_{v} n_{v}$ is not prime since $m_{v}>1$ and $n_{v}>1$, and therefore $f\left(m_{v} n_{v}\right)=0$. Thus $f\left(m_{v} n_{v}\right)=f\left(m_{v}\right) f\left(n_{v}\right)$ and $\lim _{v \rightarrow \infty} \alpha_{f}\left(m_{v}, n_{v}\right)=0$ as $m_{v} n_{v} \rightarrow \infty$ with $\left(m_{v}, n_{v}\right)$ in $\mathscr{B}^{\prime}-\mathscr{B}$.

It follows that $f \in N\left(\mathscr{B}^{\prime}\right)$ and the proof that $N(f)$ has no maximal members is complete. 


\section{REFERENCES}

(1) D. L. Goldsmtrh, On the multiplicative properties of arithmetic functions, Pacific J. Math. 27 (1968), 283-304.

(2) D. L. Goldsmith, A note on sequences of almost-multiplicative arithmetic functions, Rendiconti di Matematica 3 (6) (1970), 167-170.

Western Michigan UNIVERSITY

KaLAMAZOO

MichigAN 49001 